\title{
铜催化羧酸与芳氨基甲酰氯的脱羧交叉偶联
}

\author{
周敦樊爱红李翔陈春霞* 孙鹏彭进松* \\ (东北林业大学化学化工与资源利用学院＼cjkstart哈尔滨 150040)
}

\begin{abstract}
摘要 报道了在不加碱的条件下, 无配体铜盐能有效催化羧酸和氨基甲酰氯脱羧交叉偶联反应, 以 $\mathrm{CuCl}_{2}$ 为催化剂、苯 为溶剂, 于 $120{ }^{\circ} \mathrm{C}$ 条件下反应 $48 \mathrm{~h}$ 即可实现脱羧交叉偶联过程. 在此标准条件下, 该催化体系对官能团普适性较好, 以较高收率获得了各种酰胺, 产物经 ${ }^{1} \mathrm{H}$ NMR、 ${ }^{13} \mathrm{C}$ NMR 和 HRMS 表征

关键词＼cjkstart铜催化; 脱羒交叉偶联; 碳-碳成键; 芳氨基甲酰氯; 酰胺
\end{abstract}

\section{Copper-Catalyzed Decarboxylative Cross-Coupling of Carboxylic Acids and Arylcarbamoyl Chlorides}

\author{
Zhou, Dun Fan, Aihong Li, Xiang Chen, Chunxia* Sun, Peng Peng, Jinsong* \\ (College of Chemistry, Chemical Engineering and Resource Utilization, Northeast Forestry University, Harbin 150040)
}

\begin{abstract}
A ligand-free copper-catalyzed decarboxylative cross-coupling reaction of carboxylic acids and carbamoyl chlorides in the absence of base was developed. With $\mathrm{CuCl}_{2}$ as the catalyst, the decarboxylative cross-coupling process could be realized in benzene at $120{ }^{\circ} \mathrm{C}$ in $48 \mathrm{~h}$. Under the standard condition, the catalytic system had good functional group tolerance, and diverse amides were obtained in good to high yields. The structures of products were elucidated by ${ }^{1} \mathrm{H}$ NMR, ${ }^{13} \mathrm{C}$ NMR and HRMS spectra.

Keywords copper-catalysis; decarboxylative cross-coupling; $\mathrm{C}-\mathrm{C}$ bond formation; arylcarbamoyl chloride; amide
\end{abstract}

\section{Introduction}

Amides are a highly important class of compounds with a wide variety of applications in biochemistry, pharmacy and material science. They are usually prepared through the condensation of carboxylic acids or derivatives and amines. ${ }^{[1]}$ An alternative and less used approach to amides is the cross-coupling of organometallic reagents and carbamoyl chlorides (Scheme 1, a, b). ${ }^{[2]}$ For example, Jousseaume et $a l^{[3 \mathrm{a}]}$ reported the first palladium-catalyzed cross-coupling reaction between aryl- and vinyl-organotins and carbamoyl chlorides to give amide derivatives. Nicatalyzed cross-coupling reactions of carbamoyl chlorides and Grignard reagents were developed for the synthesis of tertiary amides by Lemoucheux et $a .^{[4]}$ The palladium-catalyzed cross-coupling reactions between organozinc and carbamoyl chlorides also provided the corresponding amides. ${ }^{[5]}$ Using organoboronic reagents (aryl boronic esters $^{[6]}$ or acids, ${ }^{[7]}$ alkylboranes, ${ }^{[8]}$ and potassium trifluoro- (a) Transition metal-catalyzed cross-coupling using organometallic reagents

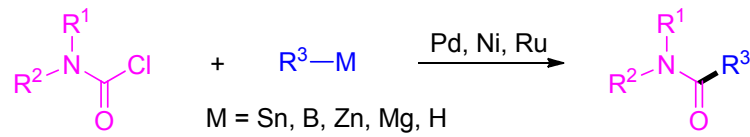

(b) Transition metal-free cross-coupling of organometallic reagents

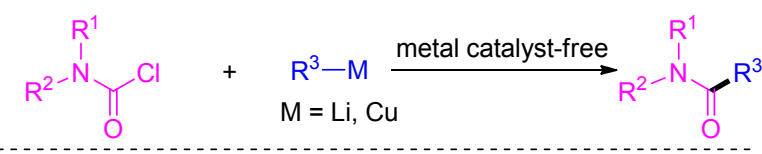

(c) This work: copper-catalyzed decarboxylative coupling

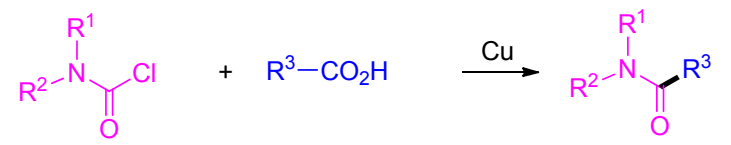

Scheme 1 Preparation of amides using carbamoyl chlorides

borates $^{[9]}$ ), Pd-catalyzed Suzuki-type reactions have been widely employed for the coupling reaction with carbamoyl

\footnotetext{
* Corresponding authors. E-mail: ccx0109@nefu.edu.cn; jspeng1998@nefu.edu.cn Received July 30, 2020; revised October 20, 2020; published online November 18, 2020.

Project supported by the Fundamental Research Funds for the Central Universities (Nos. 2572019CG06, 2572020DR07), the Natural Science Foundation of Heilongjiang Province (Nos. LC2018003, B2017002), and the Programme of Introducing Talents of Discipline to Universities (111 Project, No. B20088). 中央高校基本科研业务费专项资金(Nos. 2572019CG06, 2572020DR07)、黑龙江省自然科学基金(Nos. LC2018003, B2017002)和高等学校学科创新引智 计划(111 计划, No. B20088)资助项目.
} 
chlorides in the presence of an appropriate base. Without organometallic reagents, Kakiuchi et al. ${ }^{[10]}$ described ruthenium-catalyzed regioselective $\mathrm{C}-\mathrm{H}$ aminocarbonylation using the carbamoyl chlorides to give tertiary amides. Metal catalyst-free direct coupling of organolithiums ${ }^{[11]}$ or organocuprates $^{[12]}$ with carbamoyl chlorides is now frequently used to synthesize secondary and tertiary amides (Scheme 1, b). For example, Rouden et al ${ }^{[12]}$ described the preparation of tertiary amides via the coupling reactions of organocuprates with carbamoyl chlorides.

Organolithium, magnesium and zinc reagents were used as precursors to make the organocuprates in this reaction. Over the past decades, the carboxylic acids are promising alternatives to organic halides and organometallic reagents in organic synthesis. Transition-metal-catalyzed decarboxylative coupling reactions of carboxylic acids have emerged as powerful tools for carbon-carbon bond formation. ${ }^{[13]}$ This synthetic strategy does not use expensive and sensitive organometallic reagents, and generates $\mathrm{CO}_{2}$ instead of metal salts. Palladium, ${ }^{[14]}$ copper, ${ }^{[15]}$ silver, ${ }^{[16]}$ gold, ${ }^{[17]}$ and rhodium ${ }^{[18]}$ are capable of inducing the decarboxylation of carboxylic acids to generate organometallic species in situ, which can be utilized in various crosscoupling reactions. Inspired by Liu's ${ }^{[19]}$ report on the decarboxylative cross-coupling of potassium polyfluorobenzoates with aryl halides catalyzed by a $\mathrm{CuI} / \mathrm{Phen}$ system, herein, we are intrigued by ligand-free copper-catalyzed direct $\mathrm{C}-\mathrm{C}$ coupling of carboxylic acids with carbamoyl chlorides in the absence of base and set out to explore this possibility (Scheme 1, c).

\section{Results and discussion}

Using carbamoyl chloride 1a as the coupling partner, the coupling of acetic acid 2a instead of acetate salts was examined (Table 1). The blank experiment (without the catalyst) was examined in benzene at $80{ }^{\circ} \mathrm{C}$ for $24 \mathrm{~h}$, and no desired product 3aa was obtained. It was found that 3aa was formed in $32 \%$ yield in the presence of $15 \mathrm{~mol} \%$ $\mathrm{Cu}(\mathrm{OAc})_{2}$ in benzene at $80{ }^{\circ} \mathrm{C}$ for $24 \mathrm{~h}$ (Table 1, Entry 1).

Thus, to optimize reaction conditions, different catalysts, solvents, reaction time and temperature were examined (Table 1). The effect of copper sources [such as $\mathrm{Cu}(\mathrm{OAc})_{2}$, $\mathrm{CuCl}_{2}, \mathrm{CuI}, \mathrm{CuBr}, \mathrm{CuCl}, \mathrm{CuO}$, and $\left.\mathrm{Cu}(\mathrm{OTf})_{2}\right]$ on the reaction was first investigated, divalent $\mathrm{CuCl}_{2}$ was found to give the best result ( $53 \%$ yield, Entry 2), and $\mathrm{CuI}$ gave no product (Entry 3). A higher ratio of $\mathrm{CuCl}_{2}$ to $\mathbf{1 a}$ is preferable to afford a better yield [25\% (5 mol\%), 30\% (10 $\mathrm{mol} \%$ ), and 53\% (15 mol\%); Entries 2, 6 and 7]. Secondly, a survey of reaction media showed that non-polar solvents (benzene, $o$-xylene, $m$-xylene and xylene, Entries 8, 9, 11 , and 13) generally provided better yields than weak or strong polar solvents [tetrahydrofuran (THF), acetonitrile, $N, N$-dimethylformamide (DMF), and dimethyl sulfoxide (DMSO), Entries 10, and 14 16). Finally, a series of experiments were conducted to reveal the role of reaction time (Entries 17 and 18) and temperature (Entries 2 and
13) on the reaction. Generally, higher yields were obtained with the reaction time and temperature increasing. In general, $\mathrm{CuCl}_{2}(15 \mathrm{~mol} \%)$ was found to be the best catalyst for decarboxylative cross-coupling reaction of carboxylic acids and carbamoyl chlorides in benzene at $120{ }^{\circ} \mathrm{C}$ for $48 \mathrm{~h}$.

Table 1 Optimization of Cu-catalyzed decarboxylative coupling $^{a}$

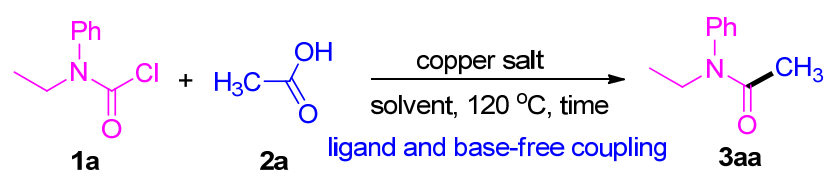

\begin{tabular}{|c|c|c|c|c|}
\hline Entry & $\mathrm{Cu}$ & Solvent & Time/h & Yield $^{b} / \%$ \\
\hline $1^{c}$ & $\mathrm{Cu}(\mathrm{OAc})_{2}$ & Benzene & 24 & 32 \\
\hline $2^{c}$ & $\mathrm{CuCl}_{2}$ & Benzene & 24 & 53 \\
\hline $3^{c}$ & $\mathrm{CuI} / \mathrm{CuBr} / \mathrm{CuCl}$ & Benzene & 24 & 0 \\
\hline $4^{c}$ & $\mathrm{CuO}$ & Benzene & 24 & 0 \\
\hline $5^{c}$ & $\mathrm{Cu}(\mathrm{OTf})_{2}$ & Benzene & 24 & 0 \\
\hline $6^{c, d}$ & $\mathrm{CuCl}_{2}$ & Benzene & 24 & 25 \\
\hline $7^{c, e}$ & $\mathrm{CuCl}_{2}$ & Benzene & 24 & 30 \\
\hline 8 & $\mathrm{CuCl}_{2}$ & $o$-Xylene & 24 & 38 \\
\hline 9 & $\mathrm{CuCl}_{2}$ & $m$-Xylene & 24 & 11 \\
\hline 10 & $\mathrm{CuCl}_{2}$ & THF & 24 & Trace \\
\hline 11 & $\mathrm{CuCl}_{2}$ & Xylene & 24 & 60 \\
\hline 12 & $\mathrm{CuCl}_{2}$ & Toluene & $24 / 48$ & Trace \\
\hline 13 & $\mathrm{CuCl}_{2}$ & Benzene & 24 & 69 \\
\hline 14 & $\mathrm{CuCl}_{2}$ & $\mathrm{CH}_{3} \mathrm{CN}$ & 24 & Trace \\
\hline 15 & $\mathrm{CuCl}_{2}$ & DMF & 24 & 0 \\
\hline 16 & $\mathrm{CuCl}_{2}$ & DMSO & 24 & Trace \\
\hline 17 & $\mathrm{CuCl}_{2}$ & Benzene & 36 & 79 \\
\hline 18 & $\mathrm{CuCl}_{2}$ & Benzene & 48 & 81 \\
\hline
\end{tabular}

${ }^{a}$ Conditions: 1a $(0.2 \mathrm{mmol})$, 2a $(0.4 \mathrm{mmol})$, catalyst $(15 \mathrm{~mol} \%)$, and solvent $(1.0 \mathrm{~mL}), 120{ }^{\circ} \mathrm{C}, 24 \mathrm{~h}^{b}{ }^{b}$ Isolated yield. ${ }^{c} 80{ }^{\circ} \mathrm{C} .{ }^{d} 5 \mathrm{~mol} \%$ of catalyst. ${ }^{e} 10$ $\mathrm{mol} \%$ of catalyst.

With the optimized reaction conditions in hand, the scope and limitations of this decarboxylative $\mathrm{C}-\mathrm{C}$ coupling reaction was then explored, a variety of diversely substituted amides 3 were obtained (Tables 2 and 3). Firstly, the generality of carboxylic acids was investigated in the process (Table 2). It was found that aliphatic carboxylic acids $(\mathbf{2} \mathbf{a} \sim \mathbf{2} \mathbf{c}), 2$-arylacetic acids $(\mathbf{2} \mathbf{d} \sim \mathbf{2} \mathbf{j})$, 3 -phenylpropiolic acid $(\mathbf{2 k})$, benzoic acids $(\mathbf{2 l} \sim \mathbf{2 n})$ and heteroaromatic acid (2o) could be converted into the amides 3 in moderate to good yields $(38 \% \sim 81 \%)$. However, when isonicotinic acid $\mathbf{2 p}$ was used, the reaction could not proceed to give the corresponding product 3ap. For aliphatic carboxylic acids, the length of the carbon chain (from C3 to C14, 3ab and 3ac) seemed not have any effect on the yield. The standard conditions were compatible with various groups such as methyl, trifluoromethyl, fluorine, chlorine, and nitro functionality at the ortho-, meta-, and para-position of the aromatic ring. In general, both the para- and meta-substituted aromatic carboxylic acids afforded a superior yield than ortho-substituted analogues 
Table 2 Variation of the carboxylic acids ${ }^{a, b}$

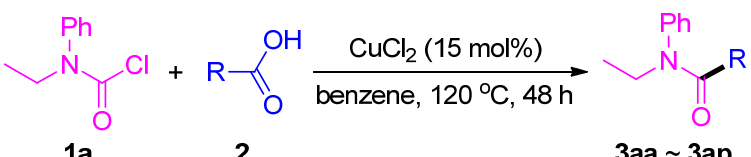

$$
\begin{aligned}
& \text { 政 }
\end{aligned}
$$

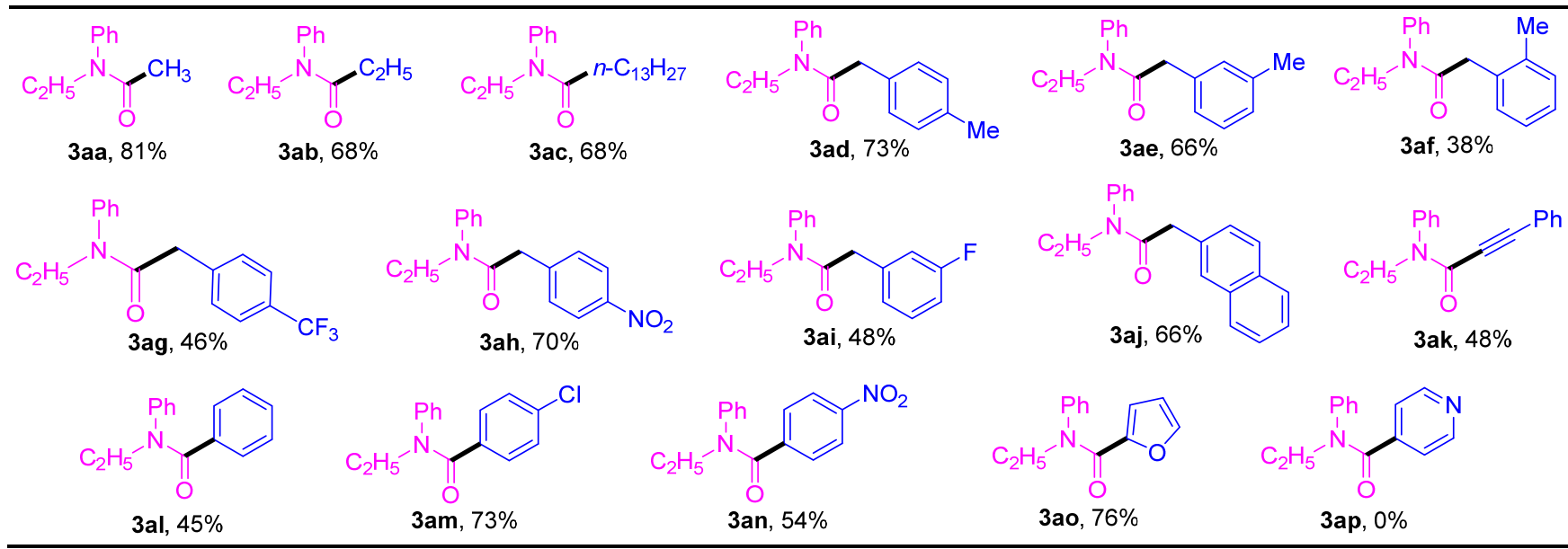

${ }^{a}$ Reaction conditions: 1a $(0.2 \mathrm{mmol})$, carboxylic acid $2(0.4 \mathrm{mmol}), 15 \mathrm{~mol} \%$ of $\mathrm{CuCl}_{2}$, benzene $(1.0 \mathrm{~mL}), 120{ }^{\circ} \mathrm{C}, 48 \mathrm{~h} .{ }^{b}$ Isolated yield.

Table 3 Variation of the arylcarbamoyl chlorides ${ }^{a, b}$

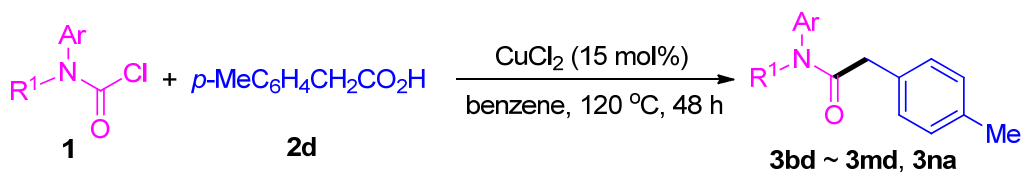

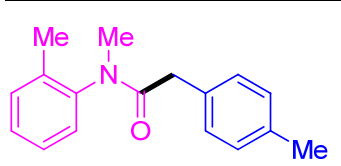

3bd, $64 \%$

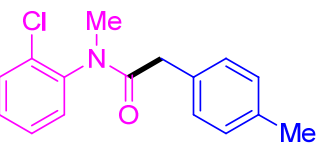

$3 \mathrm{fd}, 35 \%$<smiles>Cc1ccc(CC(=O)N(C)c2cccc(C)c2)cc1</smiles>

3 cd, $50 \%$<smiles>Cc1ccc(CC(=O)N(C)c2ccc(C)cc2)cc1</smiles>

3dd, $81 \%$<smiles>COc1ccc(N(C)C(=O)Cc2ccc(C)cc2)cc1</smiles>

3ed, $89 \%$<smiles>Cc1ccc(CC(=O)N(C)c2ccc(C#N)cc2)cc1</smiles>

3id, $64 \%$<smiles>CN(C(=O)Cc1ccc([N+](=O)[O-])cc1)c1ccc2ccccc2c1</smiles>

3jd, 75\%<smiles>Cc1ccc(CC(=O)N(C)c2ccc(Cl)cc2)cc1</smiles>

3gd, $63 \%$<smiles>Cc1ccc(CC(=O)N(C)c2ccc(C(F)(F)F)cc2)cc1</smiles>

3hd, $57 \%$<smiles>CC(=O)N(C)c1ccccc1</smiles>

$3 a^{c}, 68 \%$<smiles>O=C(c1ccccc1)N1CCOCC1</smiles>

3ol, $76 \%$

$\overline{{ }^{a}}$ Reaction conditions: arylcarbamoyl chlorides $1(0.2 \mathrm{mmol}), \mathbf{2 d}(0.4 \mathrm{mmol}), 15 \mathrm{~mol} \%$ of $\mathrm{CuCl}_{2}$, benzene $(1.0 \mathrm{~mL}), 120{ }^{\circ} \mathrm{C}, 48 \mathrm{~h} .{ }^{b}$ Isolated yield. ${ }^{c}$ Acetic acid was used.

(for example, 3af, 38\% yield) as the result of the steric hindrance of substituents.

Secondly, the scope of arylcarbamoyl chlorides was then explored using $p$-tolyl acetic acid $\mathbf{2 d}$ as the coupling partner (Table 3 ). A variety of substituents on the aryl moiety were applicable, affording the corresponding products 3bd $\sim 3 \mathbf{m d}$ in $35 \% \sim 89 \%$ yields (Table 3 ). Both electrondonating $(\mathrm{Me}, \mathrm{OMe})$ and -withdrawing groups $\left(\mathrm{CF}_{3}, \mathrm{Cl}\right.$, and $\mathrm{CN}$ ) were tolerant under the reaction conditions. The electronic nature of aniline skeleton seemed to affect the efficiency to some extent, arylcarbamoyl chlorides con- taining electron-donating groups underwent the reaction smoothly and afforded good yields (3bd 3ed, 50\% $89 \%$ ). $\quad N$-Methyl arylcarbamoyl chlorides containing groups at the para-position of aniline (1d, 1g) were generally more reactive than those bearing substituents at the ortho- or meta-position (1b, 1c, and 1f) and provided higher yields. In addition, $\beta$-naphthylcarbamoyl chloride could also be transformed into the desired products $\mathbf{3 j d}$ in $75 \%$ yield. Phenylcarbamoyl chlorides $1 \mathbf{k} \sim \mathbf{1 n}$ were finally investigated to elucidate the influence of different $N$-substituted groups (methyl, benzyl, cyclohexyl, and 
phenyl) on the reaction. $N$-Aliphatic substituted substrates generally provided high yields $(68 \% \sim 85 \%)$, however, the introduction of the aromatic substituent on the $\mathrm{N}$ atom (for example, dipheylcarbamoyl chloride 1m) decreased reactivity and the product 3md was obtained in $43 \%$ yield. Two alkyl substituted carbamoyl chloride has been examined, for example, the decarboxylative $\mathrm{C}-\mathrm{C}$ coupling reaction of morpholine-4-carbonyl chloride and benzoic acid gave the corresponding product $\mathbf{3 o l}$ in $76 \%$ yield.

To gain insight into the mechanism of the reaction, 2,2,6,6-tetramethylpiperidinooxy (TEMPO) as a radical probe was added to the reaction of morpholine-4-carbonyl chloride and benzoic acid, the decarboxylation step was not blocked and gave the product 301 in $72 \%$ yield. On the basis of the above results, a plausible pathway was proposed for this $\mathrm{Cu}$ (II)-catalyzed decarboxylative $\mathrm{C}-\mathrm{C}$ coupling reaction. Scheme 2 shows a simplified sequence of events beginning with the active $\mathrm{Cu}$ (II) catalyst. The extrusion of $\mathrm{HCl}$ took place between carboxylic acid $\mathbf{2}$ and $\mathrm{CuCl}_{2}$ to give copper(II) carboxylate 4 , which would undergo the decarboxylative reaction to afford organo-copper species $\mathbf{5}$ with nucleophilic reactivity via the extrusion of carbon dioxide. The carbon dioxide and $\mathrm{HCl}$ generated from the above process were detected by headspace gas chromatography (HS-GC). The subsequent step involved the coordination of arylcarbamoyl chloride $\mathbf{1}$ to the organo- $\mathrm{Cu}$ (II) center to form $\mathbf{6}$, which was followed by a nucleophilic addition of organo-copper species across the $\mathrm{C}=\mathrm{O}$ bond to provide an intermediate complex 7 . $\beta$-Chlorine elimination afforded the amide $\mathbf{3}$ and regenerated the $\mathrm{Cu}(\mathrm{II})$ catalyst.

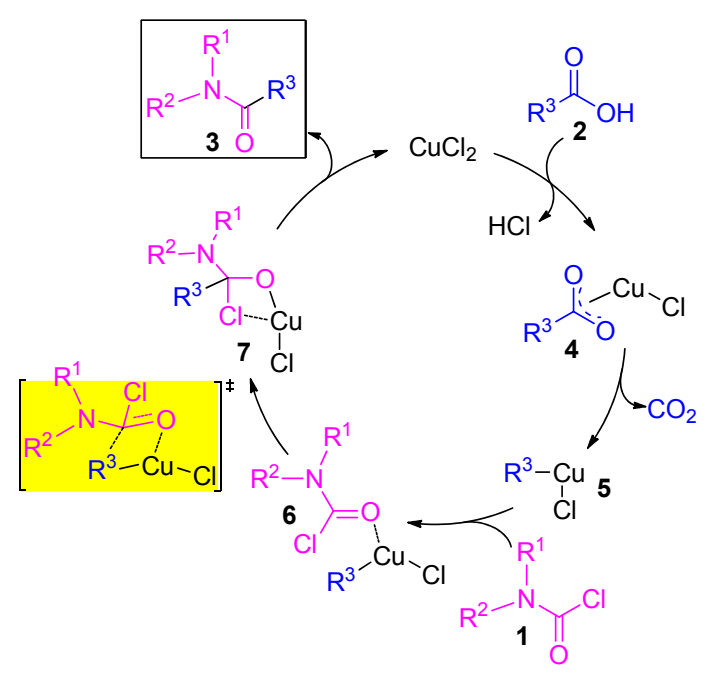

Scheme 2 Proposed catalytic pathway

\section{Conclusions}

In summary, a ligand-free $\mathrm{Cu}(\mathrm{II})$-catalyzed decarboxylation reaction that can use directly different types of carboxylic acids has been developed. Under the mild condition, a broad range of functional groups is compatible with this $\mathrm{C}-\mathrm{C}$ coupling reaction, and diverse amides can be obtained in good to high yields.

\section{Experimental section}

\subsection{General experimental methods}

Melting points were determined on a melting point apparatus in open capillaries and uncorrected. Infrared spectra of samples were recorded from 4000 to $500 \mathrm{~cm}^{-1}$ in ATR (attenuated total reflectance) mode using an FT-IR instrument. ${ }^{1} \mathrm{H}$ NMR spectra were recorded on a $500 \mathrm{MHz}$ spectrometer, and ${ }^{13} \mathrm{C}$ NMR spectra were recorded at 126 $\mathrm{MHz}$. Unless otherwise stated, deuterochloroform $\left(\mathrm{CDCl}_{3}\right)$ was used as a solvent. Chemical shifts $(\delta)$ are given relative to tetramethylsilane (TMS, $\left.\delta_{\mathrm{H}} 0\right)$ or $\mathrm{CHCl}_{3}\left(\delta_{\mathrm{C}} 77.16\right)$. High-resolution mass spectra were recorded on a BIO TOF $\mathrm{Q}$ mass spectrometer equipped with an electrospray ion source (ESI), and operated in the positive mode.

Chemicals were all purchased from commercial supplies and used without further purification unless otherwise stated. Solvents were dried and purified according to the standard procedures before use. Reactions were monitored by analytical thin-layer chromatography (TLC). All reactions were conducted in dried glassware. Purification of reaction products was done by flash chromatography with 230 400 mesh silica gel. All the arylcarbamoyl chloride substrates were prepared according to literature methods. $^{[20]}$

\subsection{General procedure for Copper-catalyzed de-} carboxylative coupling

A $10 \mathrm{~mL}$ vial equipped with a magnetic stirring bar was charged with arylcarbamoyl chloride 1 (0.2 mmol, 1.0 equiv.), carboxylic acid 2 (0.4 mmol, 2.0 equiv.), and then $\mathrm{CuCl}_{2}(0.03 \mathrm{mmol}, 4.0 \mathrm{mg})$ were added. Finally, benzene $(1.0 \mathrm{~mL})$ was added to the mixture via syringe at room temperature under air. The tube was sealed and put into a preheated oil bath at $120{ }^{\circ} \mathrm{C}$ for $48 \mathrm{~h}$. The mixture was cooled to room temperature, quenched with water $(5 \mathrm{~mL})$, and diluted with ethyl acetate $(5 \mathrm{~mL})$. The layers were separated, and the aqueous layer was extracted with ethyl acetate $(5 \mathrm{~mL} \times 2)$. The combined organic extracts were dried over anhydrous magnesium sulfate, filtered, and concentrated in vacuo. The crude product was then purified by flash chromatography on silica gel $(\mathrm{H})$ with ethyl acetate/petroleum ether $(V: V=1: 3)$ as eluent. $N$-Ethyl$N$-phenylacetamide (3aa): Yield $81 \%(26.1 \mathrm{mg})$, yellow solid. m.p. 55 56 ${ }^{\circ} \mathrm{C}$ (lit. ${ }^{[21]} 55{ }^{\circ} \mathrm{C}$ ); ${ }^{1} \mathrm{H}$ NMR $(500 \mathrm{MHz}$, $\left.\mathrm{CDCl}_{3}\right) \delta: 7.40(\mathrm{t}, J=7.8 \mathrm{~Hz}, 2 \mathrm{H}), 7.33(\mathrm{t}, J=7.4 \mathrm{~Hz}, 1 \mathrm{H})$, $7.16 \sim 7.13(\mathrm{~m}, 2 \mathrm{H}), 3.73(\mathrm{q}, J=7.2 \mathrm{~Hz}, 2 \mathrm{H}), 1.80(\mathrm{~s}, 3 \mathrm{H})$, 1.09 (t, $J=7.1 \mathrm{~Hz}, 3 \mathrm{H}) ;{ }^{13} \mathrm{C} \mathrm{NMR}\left(126 \mathrm{MHz}, \mathrm{CDCl}_{3}\right) \delta$ : $169.9,142.9,129.7,128.2,127.8,43.8,22.8,13.1$; IR (KBr) v: 2980, 1642, 1494, 1401, 1297, 768, $709 \mathrm{~cm}^{-1}$.

$N$-Ethyl- $N$-phenylpropionamide (3ab): ${ }^{[22]}$ Yield $68 \%$ (24.1 mg), yellow liquid. ${ }^{1} \mathrm{H}$ NMR $\left(500 \mathrm{MHz}, \mathrm{CDCl}_{3}\right) \delta$ : $7.40(\mathrm{t}, J=7.5 \mathrm{~Hz}, 2 \mathrm{H}), 7.33(\mathrm{t}, J=7.2 \mathrm{~Hz}, 1 \mathrm{H}), 7.16 \sim 7.12$ $(\mathrm{m}, 2 \mathrm{H}), 3.74(\mathrm{q}, J=7.1 \mathrm{~Hz}, 2 \mathrm{H}), 2.01(\mathrm{q}, J=7.5 \mathrm{~Hz}, 2 \mathrm{H})$, 1.09 (t, $J=7.1 \mathrm{~Hz}, 3 \mathrm{H}), 1.02$ (t, $J=7.3 \mathrm{~Hz}, 3 \mathrm{H}) ;{ }^{13} \mathrm{C} \mathrm{NMR}$ $\left(126 \mathrm{MHz}, \mathrm{CDCl}_{3}\right) \delta: 172.4,141.5,128.6,127.4,126.8$, 
42.9, 26.9, 12.1, 8.6; IR (KBr) v: 2970, 1658, 1496, 1404, $1261,702 \mathrm{~cm}^{-1}$.

$N$-Ethyl- $N$-phenyltetradecanamide (3ac): Yield 68\% (45.0 mg), light yellow liquid. ${ }^{1} \mathrm{H}$ NMR (500 MHz, $\mathrm{CDCl}_{3}$ ) $\delta: 7.39(\mathrm{t}, J=7.5 \mathrm{~Hz}, 2 \mathrm{H}), 7.32(\mathrm{t}, J=7.5 \mathrm{~Hz}, 1 \mathrm{H}), 7.12$ (d, $J=7.7 \mathrm{~Hz}, 2 \mathrm{H}), 3.72(\mathrm{q}, J=7.2 \mathrm{~Hz}, 2 \mathrm{H}), 1.98(\mathrm{t}, J=7.6$ $\mathrm{Hz}, 2 \mathrm{H}), 1.54 \sim 1.51(\mathrm{~m}, 2 \mathrm{H}), 1.30 \sim 1.11(\mathrm{~m}, 20 \mathrm{H}), 1.08$ (t, $J=7.3 \mathrm{~Hz}, 3 \mathrm{H}), 0.85$ (t, $J=6.8 \mathrm{~Hz}, 3 \mathrm{H}) ;{ }^{13} \mathrm{C}$ NMR $(126$ $\left.\mathrm{MHz}, \mathrm{CDCl}_{3}\right) \delta: 171.8,141.6,128.6,127.4,126.8,42.9$, $33.5,30.9,28.7,28.6,28.5,28.4,28.34,28.30,28.25,24.5$, 21.7, 13.1, 12.1; IR (KBr) v: 2925, 1662, 1496, 1261, 768 $\mathrm{cm}^{-1}$; HRMS-ESI calcd for $\mathrm{C}_{22} \mathrm{H}_{37} \mathrm{NONa}[\mathrm{M}+\mathrm{Na}]^{+}$ 354.27674, found 354.27679.

$N$-Ethyl- $N$-phenyl-2-( $p$-tolyl)acetamide (3ad) $:^{[23]}$ Yield $73 \%$ (36.9 mg), light yellow liquid. ${ }^{1} \mathrm{H}$ NMR (500 MHz, $\left.\mathrm{CDCl}_{3}\right) \delta: 7.41 \sim 7.34(\mathrm{~m}, 3 \mathrm{H}), 7.09(\mathrm{~d}, J=7.0 \mathrm{~Hz}, 2 \mathrm{H})$, 7.04 (d, $J=7.6 \mathrm{~Hz}, 2 \mathrm{H}), 6.94$ (d, $J=7.7 \mathrm{~Hz}, 2 \mathrm{H}), 3.75$ (q, $J=7.2 \mathrm{~Hz}, 2 \mathrm{H}), 3.36(\mathrm{~s}, 2 \mathrm{H}), 2.30(\mathrm{~s}, 3 \mathrm{H}), 1.10$ (t, $J=7.2$ $\mathrm{Hz}, 3 \mathrm{H}) ;{ }^{13} \mathrm{C}$ NMR $\left(126 \mathrm{MHz}, \mathrm{CDCl}_{3}\right) \delta: 169.5,141.3$, $135.0,131.4,128.5,127.9,127.9,127.7,126.9,43.2,39.8$, 20.0, 12.0; IR (KBr) v: 3483, 2928, 1660, 1494, 1130, 769 $\mathrm{cm}^{-1}$.

$N$-Ethyl- $N$-phenyl-2-( $m$-tolyl)acetamide (3ae): Yield $66 \%$ (33.4 mg), yellow liquid. ${ }^{1} \mathrm{H}$ NMR (500 $\mathrm{MHz}, \mathrm{CDCl}_{3}$ ) $\delta: 7.44 \sim 7.32(\mathrm{~m}, 3 \mathrm{H}), 7.19 \sim 7.01(\mathrm{~m}, 6 \mathrm{H}), 3.78(\mathrm{q}, J=$ $7.2 \mathrm{~Hz}, 2 \mathrm{H}), 3.40(\mathrm{~s}, 2 \mathrm{H}), 2.02(\mathrm{~s}, 3 \mathrm{H}), 1.14(\mathrm{t}, J=7.2 \mathrm{~Hz}$, $3 \mathrm{H}) ;{ }^{13} \mathrm{C}$ NMR $\left(126 \mathrm{MHz}, \mathrm{CDCl}_{3}\right) \delta: 169.4,141.3,135.4$, $133.4,129.0,128.6,128.4,127.5,127.0,125.7,124.8$, 43.3, 38.2, 18.4, 12.0; IR (KBr) v: 3490, 2971, 1660, 1494, $1131,746 \mathrm{~cm}^{-1}$; HRMS-ESI calcd for $\mathrm{C}_{17} \mathrm{H}_{19} \mathrm{NONa}[\mathrm{M}+$ $\mathrm{Na}]^{+}$276.13589, found 276.13538.

$N$-Ethyl- $N$-phenyl-2-(o-tolyl)acetamide (3af): Yield $38 \%$ (19.3 mg), light red liquid. ${ }^{1} \mathrm{H}$ NMR $(500 \mathrm{MHz}$, $\left.\mathrm{CDCl}_{3}\right) \delta: 7.41 \sim 7.34(\mathrm{~m}, 3 \mathrm{H}), 7.14 \sim 7.04(\mathrm{~m}, 6 \mathrm{H}), 3.78$ (q, $J=7.2 \mathrm{~Hz}, 2 \mathrm{H}), 3.38(\mathrm{~s}, 2 \mathrm{H}), 2.02(\mathrm{~s}, 3 \mathrm{H}), 1.14$ (t, $J=$ $7.2 \mathrm{~Hz}, 3 \mathrm{H}) ;{ }^{13} \mathrm{C}$ NMR $(126 \mathrm{MHz}, \mathrm{CDCl} 3) \delta: 169.4,141.3$, $135.4,133.4,129.0,128.6,128.4,127.5,127.0,125.7$, $124.9,43.3,38.2,18.4,12.1$; IR (KBr) v: 3482, 2970, 1659, 1494, 1131, $746 \mathrm{~cm}^{-1}$; HRMS-ESI calcd for $\mathrm{C}_{17} \mathrm{H}_{19} \mathrm{NONa}[\mathrm{M}+\mathrm{Na}]^{+}$276.13589, found 276.13550.

$N$-Ethyl- $N$-phenyl-2-(4-(trifluoromethyl)phenyl)acetamide (3ag): Yield 46\% (28.2 mg), light yellow liquid. ${ }^{1} \mathrm{H}$ NMR $\left(500 \mathrm{MHz}, \mathrm{CDCl}_{3}\right) \delta: 7.48(\mathrm{~d}, J=8.1 \mathrm{~Hz}, 2 \mathrm{H})$, $7.44 \sim 7.37(\mathrm{~m}, 3 \mathrm{H}), 7.17(\mathrm{~d}, J=7.9 \mathrm{~Hz}, 2 \mathrm{H}), 7.12 \sim 7.07$ (m, 2H), 3.76 (q, $J=7.1 \mathrm{~Hz}, 2 \mathrm{H}), 3.45(\mathrm{~s}, 2 \mathrm{H}), 1.11(\mathrm{t}, J=$ $7.1 \mathrm{~Hz}, 3 \mathrm{H}) ;{ }^{13} \mathrm{C}$ NMR $\left(126 \mathrm{MHz}, \mathrm{CDCl}_{3}\right) \delta: 168.4,141.0$, 138.6, 128.7, 128.4, 127.8 (128.2, 128.0, 127.7, 127.5, q, $\left.{ }^{2} J_{\mathrm{C}-\mathrm{F}}=32 \mathrm{~Hz}\right), \quad 127.6,127.2,124.1 \quad(124.17,124.14$, 124.11, 124.08, q, $\left.{ }^{3} J_{\mathrm{C}-\mathrm{F}}=4 \mathrm{~Hz}\right), 123.2(126.5,124.3$, $\left.122.1,120.0, \mathrm{q},{ }^{1} J_{\mathrm{C}-\mathrm{F}}=272 \mathrm{~Hz}\right), 43.4,40.1,11.9$; IR $(\mathrm{KBr})$ $v: 2972,1659,1495,1326,1126,1067,701 \mathrm{~cm}^{-1}$; HRMS-ESI calcd for $\mathrm{C}_{17} \mathrm{H}_{16} \mathrm{~F}_{3} \mathrm{NONa}[\mathrm{M}+\mathrm{Na}]$ 330.10762 , found 330.10773 .

$N$-Ethyl-2-(4-nitrophenyl)- $N$-phenylacetamide (3ah): ${ }^{[23]}$ Yield 70\% (39.8 mg), red oil. IR (KBr) v: 3490, 2974, 1659, 1346, 856, 732, $560 \mathrm{~cm}^{-1}$; ${ }^{1} \mathrm{H}$ NMR $(500 \mathrm{MHz}$, $\left.\mathrm{CDCl}_{3}\right) \delta: 8.15 \sim 8.02(\mathrm{~m}, 2 \mathrm{H}), 7.48 \sim 7.34(\mathrm{~m}, 3 \mathrm{H}), 7.22$ (d, $J=8.6 \mathrm{~Hz}, 2 \mathrm{H}), 7.10(\mathrm{~d}, J=7.6 \mathrm{~Hz}, 2 \mathrm{H}), 3.76$ (q, $J=$ $7.1 \mathrm{~Hz}, 2 \mathrm{H}), 3.49$ (s, 2H), $1.11(\mathrm{t}, J=7.1 \mathrm{~Hz}, 3 \mathrm{H}) ;{ }^{13} \mathrm{C}$ NMR $\left(126 \mathrm{MHz}, \mathrm{CDCl}_{3}\right) \delta: 167.8,145.8,142.1,140.8$, 129.1, 128.9, 127.5, 127.4, 122.4, 43.4, 40.2, 11.9 .

$N$-Ethyl-2-(3-fluorophenyl)- $N$-phenylacetamide (3ai): Yield 48\% (24.7 mg), light yellow liquid. ${ }^{1} \mathrm{H}$ NMR (500 $\left.\mathrm{MHz}, \mathrm{CDCl}_{3}\right) \delta: 7.44 \sim 7.33(\mathrm{~m}, 3 \mathrm{H}), 7.17(\mathrm{q}, J=7.8 \mathrm{~Hz}$, $1 \mathrm{H}), 7.08(\mathrm{~d}, J=6.9 \mathrm{~Hz}, 2 \mathrm{H}), 6.87(\mathrm{td}, J=8.5,2.1 \mathrm{~Hz}$, $1 \mathrm{H}), 6.81(\mathrm{~d}, J=7.6 \mathrm{~Hz}, 1 \mathrm{H}), 6.77(\mathrm{~d}, J=9.9 \mathrm{~Hz}, 1 \mathrm{H}), 3.75$ (q, $J=7.1 \mathrm{~Hz}, 2 \mathrm{H}), 3.39$ (s, 2H), 1.11 (t, $J=7.2 \mathrm{~Hz}, 3 \mathrm{H})$; ${ }^{13} \mathrm{C}$ NMR $\left(126 \mathrm{MHz}, \mathrm{CDCl}_{3}\right) \delta: 168.7,161.6$ (162.6, 160.7, $\left.\mathrm{d},{ }^{1} J_{\mathrm{C}-\mathrm{F}}=246 \mathrm{~Hz}\right), 141.0,136.9\left(136.91,136.85, \mathrm{~d},{ }^{3} J_{\mathrm{C}-\mathrm{F}}=\right.$ $8 \mathrm{~Hz}), 128.6,128.5,127.6,127.1,123.7$ (123.72, 123.70, $\left.\mathrm{d},{ }^{4} J_{\mathrm{C}-\mathrm{F}}=3 \mathrm{~Hz}\right), 115.0\left(115.1,114.9, \mathrm{~d},{ }^{2} J_{\mathrm{C}-\mathrm{F}}=21 \mathrm{~Hz}\right)$, $112.4\left(112.5,112.3, \mathrm{~d},{ }^{2} J_{\mathrm{C}-\mathrm{F}}=20 \mathrm{~Hz}\right), 43.3,40.0\left(\mathrm{~d},{ }^{4} J_{\mathrm{C}-\mathrm{F}}=\right.$ $1 \mathrm{~Hz}$ ), 12.0. IR (KBr) v: 2973, 1657, 1593, 1267, 1132, 769, $702 \mathrm{~cm}^{-1}$; HRMS-ESI calcd for $\mathrm{C}_{16} \mathrm{H}_{16} \mathrm{FNONa}[\mathrm{M}+$ $\mathrm{Na}]^{+}$280.11081, found 280.11093.

$N$-Ethyl-2-(naphthalen-2-yl)- $N$-phenylacetamide (3aj): Yield 66\% (38.2 mg), light yellow liquid. ${ }^{1} \mathrm{H}$ NMR (500 $\left.\mathrm{MHz}, \mathrm{CDCl}_{3}\right) \delta: 7.72 \sim 7.66(\mathrm{~m}, 1 \mathrm{H}), 7.62(\mathrm{t}, J=7.9 \mathrm{~Hz}$, $2 \mathrm{H}), 7.38 \sim 7.31(\mathrm{~m}, 3 \mathrm{H}), 7.30 \sim 7.22(\mathrm{~m}, 3 \mathrm{H}), 7.20 \sim 7.07$ $(\mathrm{m}, 1 \mathrm{H}), 7.02 \sim 6.94(\mathrm{~m}, 2 \mathrm{H}), 3.68(\mathrm{q}, J=7.1 \mathrm{~Hz}, 2 \mathrm{H})$, 3.49 (s, 2H), 1.03 (t, $J=7.1 \mathrm{~Hz}, 3 \mathrm{H}) ;{ }^{13} \mathrm{C}$ NMR (126 MHz, $\left.\mathrm{CDCl}_{3}\right) \delta: 169.3,141.2,132.4,132.0,131.2,128.5,127.7$, $127.0,126.8,126.6,126.54,126.52,126.4,124.8,124.4$, 43.3, 40.6, 12.0; IR (KBr) v: 3482, 3055, 2971, 2932, 1654, 1494, 1129, 701, $561 \mathrm{~cm}^{-1}$; HRMS-ESI calcd for $\mathrm{C}_{20} \mathrm{H}_{19} \mathrm{NONa}[\mathrm{M}+\mathrm{Na}]^{+}$312.13589, found 312.13593.

$N$-Ethyl- $N, 3$-diphenylpropiolamide (3ak): Yield $48 \%$ (23.9 mg), white solid, m.p. $100 \sim 101{ }^{\circ} \mathrm{C}$ (lit. ${ }^{[2]} 100 \sim$ $\left.101{ }^{\circ} \mathrm{C}\right) ;{ }^{1} \mathrm{H}$ NMR $(500 \mathrm{MHz}, \mathrm{CDCl} 3) \delta: 7.39 \sim 7.28(\mathrm{~m}$, $3 \mathrm{H}), 7.26 \sim 7.17(\mathrm{~m}, 3 \mathrm{H}), 7.15 \sim 7.08(\mathrm{~m}, 2 \mathrm{H}), 7.00(\mathrm{~d}, J=$ $7.4 \mathrm{~Hz}, 2 \mathrm{H}), 3.78$ (q, $J=7.0 \mathrm{~Hz}, 2 \mathrm{H}), 1.09$ (t, $J=7.1 \mathrm{~Hz}$, $3 \mathrm{H}) ;{ }^{13} \mathrm{C} \mathrm{NMR}\left(126 \mathrm{MHz}, \mathrm{CDCl}_{3}\right) \delta: 152.9,140.7,131.4$, $128.8,128.1,127.7,127.3,127.1,119.5,89.7,81.8,42.4$, 12.0; IR (KBr) v: 2964, 2213, 1632, 1492, 1347, 1310, $1261,1025,759,700,529 \mathrm{~cm}^{-1}$.

$N$-Ethyl- $N$-phenylbenzamide (3al): ${ }^{[25]}$ Yield 73\% (20.3 $\mathrm{mg})$, black liquid. ${ }^{1} \mathrm{H}$ NMR $\left(500 \mathrm{MHz}, \mathrm{CDCl}_{3}\right) \delta: 7.20(\mathrm{t}$, $J=7.7 \mathrm{~Hz}, 2 \mathrm{H}), 7.17 \sim 7.11(\mathrm{~m}, 3 \mathrm{H}), 7.10 \sim 7.03(\mathrm{~m}, 3 \mathrm{H})$, 6.95 (d, $J=7.6 \mathrm{~Hz}, 2 \mathrm{H}), 3.91(\mathrm{q}, J=7.2 \mathrm{~Hz}, 2 \mathrm{H}), 1.15$ (t, $J=7.1 \mathrm{~Hz}, 3 \mathrm{H}) ;{ }^{13} \mathrm{C} \mathrm{NMR}\left(126 \mathrm{MHz}, \mathrm{CDCl}_{3}\right) \delta: 169.3$, $142.1,135.2,128.4,128.1,127.6,126.9,126.7,125.6$, 44.4, 11.9; IR (KBr) v: 3483, 2973, 1638, 1493, 1118, 699, $585 \mathrm{~cm}^{-1}$

4-Chloro- $N$-ethyl- $N$-phenylbenzamide (3am): ${ }^{[23]}$ Yield $73 \%$ (37.8 mg), light yellow liquid. ${ }^{1} \mathrm{H}$ NMR (500 MHz, $\left.\mathrm{CDCl}_{3}\right) \delta: 7.20 \sim 7.13(\mathrm{~m}, 4 \mathrm{H}), 7.13 \sim 7.08(\mathrm{~m}, 1 \mathrm{H}), 7.05$ $(\mathrm{d}, J=8.3 \mathrm{~Hz}, 2 \mathrm{H}), 6.94(\mathrm{~d}, J=8.0 \mathrm{~Hz}, 2 \mathrm{H}), 3.90(\mathrm{q}, J=$ $7.1 \mathrm{~Hz}, 2 \mathrm{H}), 1.14(\mathrm{t}, J=7.1 \mathrm{~Hz}, 3 \mathrm{H}) ;{ }^{13} \mathrm{C} \mathrm{NMR}(126 \mathrm{MHz}$, $\left.\mathrm{CDCl}_{3}\right) \delta: 168.0,142.0,134.5,133.7,129.2,128.3,126.93$, 126.85, 125.9, 44.5, 11.8; IR (KBr) v: 2973, 1647, 1493, $1091,754,583 \mathrm{~cm}^{-1}$.

$N$-Ethyl-4-nitro- $N$-phenylbenzamide (3an): Yield 54\% (29.2 mg), light yellow solid. m.p. 117 119 ${ }^{\circ} \mathrm{C}$ (lit. $^{[23]}$ $\left.119 \sim 120{ }^{\circ} \mathrm{C}\right) ;{ }^{1} \mathrm{H}$ NMR $\left(500 \mathrm{MHz}, \mathrm{CDCl}_{3}\right) \delta: 7.99(\mathrm{~d}$, 
$J=8.1 \mathrm{~Hz}, 2 \mathrm{H}), 7.42(\mathrm{~d}, J=7.9 \mathrm{~Hz}, 2 \mathrm{H}), 7.28 \sim 7.13(\mathrm{~m}$, $3 \mathrm{H}), 7.01$ (d, $J=7.6 \mathrm{~Hz}, 2 \mathrm{H}), 3.98$ (q, $J=7.0 \mathrm{~Hz}, 2 \mathrm{H}), 1.22$ $(\mathrm{t}, J=7.1 \mathrm{~Hz}, 3 \mathrm{H}) ;{ }^{13} \mathrm{C} \mathrm{NMR}\left(126 \mathrm{MHz}, \mathrm{CDCl}_{3}\right) \delta: 167.9$, $147.9,142.6,142.1,129.52,129.49,128.0,127.5,123.0$, $45.4,12.8$; IR (KBr) v: 2976, 1651, 1347, 846, 763, 699, $580 \mathrm{~cm}^{-1}$.

$N$-Ethyl- $N$-phenylfuran-2-carboxamide (3ao): Yield 76\% (32.7 mg), white solid. m.p. $174 \sim 177{ }^{\circ} \mathrm{C}$ (lit. ${ }^{[26]}$ $\left.175 \sim 176{ }^{\circ} \mathrm{C}\right) ;{ }^{1} \mathrm{H}$ NMR $\left(500 \mathrm{MHz}, \mathrm{CDCl}_{3}\right) \delta: 7.44 \sim 7.32$ $(\mathrm{m}, 3 \mathrm{H}), 7.30(\mathrm{~d}, J=1.0 \mathrm{~Hz}, 1 \mathrm{H}), 7.21 \sim 7.14(\mathrm{~m}, 2 \mathrm{H})$, $6.16(\mathrm{dd}, J=3.5,1.7 \mathrm{~Hz}, 1 \mathrm{H}), 5.72(\mathrm{~d}, J=2.7 \mathrm{~Hz}, 1 \mathrm{H})$, 3.90 (q, $J=7.1 \mathrm{~Hz}, 2 \mathrm{H}), 1.20(\mathrm{t}, J=7.1 \mathrm{~Hz}, 3 \mathrm{H}) ;{ }^{13} \mathrm{C} \mathrm{NMR}$ $\left(126 \mathrm{MHz}, \mathrm{CDCl}_{3}\right) \delta: 158.8,147.2,144.2,142.4,129.5$, $128.5,128.0,116.1,110.9,45.4,12.8$; IR (KBr) v: 3127, 3107, 2984, 1628, 1469, 1412, 1309, 1120, 1028, 704, 581 $\mathrm{cm}^{-1}$.

$N$-Methyl- $N$-(o-tolyl)-2-( $p$-tolyl)acetamide (3bd): Yield 64\% (32.4 mg), light yellow liquid. ${ }^{1} \mathrm{H}$ NMR (500 MHz, $\left.\mathrm{CDCl}_{3}\right) \delta: 7.23 \sim 7.13(\mathrm{~m}, 3 \mathrm{H}), 6.98(\mathrm{~d}, J=7.6 \mathrm{~Hz}, 1 \mathrm{H})$, $6.94(\mathrm{~d}, J=7.8 \mathrm{~Hz}, 2 \mathrm{H}), 6.81$ (d, $J=7.9 \mathrm{~Hz}, 2 \mathrm{H}), 3.23$ (s, 2H), $3.09(\mathrm{~s}, 3 \mathrm{H}), 2.20(\mathrm{~s}, 3 \mathrm{H}), 1.99(\mathrm{~s}, 3 \mathrm{H}) ;{ }^{13} \mathrm{C} \mathrm{NMR}$ $\left(126 \mathrm{MHz}, \mathrm{CDCl}_{3}\right) \delta: 170.3,141.5,135.1,134.8,130.9$, $130.4,128.0,128.0,127.4,126.3,39.4,35.2,20.0,16.2$; IR (KBr) v: 3482, 2923, 1657, 1493, 1376, 1103, 727, 569 $\mathrm{cm}^{-1}$; HRMS-ESI calcd for $\mathrm{C}_{17} \mathrm{H}_{20} \mathrm{NO}[\mathrm{M}+\mathrm{H}]^{+}$ 254.1539, found 254.1532.

$N$-Methyl- $N$-( $m$-tolyl)-2-( $p$-tolyl)acetamide (3cd): Yield $50 \%(25.3 \mathrm{mg})$, light yellow liquid. ${ }^{1} \mathrm{H}$ NMR (500 MHz, $\left.\mathrm{CDCl}_{3}\right) \delta: 7.19(\mathrm{t}, J=7.7 \mathrm{~Hz}, 1 \mathrm{H}), 7.07(\mathrm{~d}, J=7.6 \mathrm{~Hz}, 1 \mathrm{H})$, $6.96(\mathrm{~d}, J=7.7 \mathrm{~Hz}, 2 \mathrm{H}), 6.91 \sim 6.82(\mathrm{~m}, 3 \mathrm{H}), 6.80(\mathrm{~s}, 1 \mathrm{H})$, $3.32(\mathrm{~s}, 2 \mathrm{H}), 3.16(\mathrm{~s}, 3 \mathrm{H}), 2.26(\mathrm{~s}, 3 \mathrm{H}), 2.21(\mathrm{~s}, 3 \mathrm{H}) ;{ }^{13} \mathrm{C}$ NMR $\left(126 \mathrm{MHz}, \mathrm{CDCl}_{3}\right) \delta: 170.2,142.9,138.6,135.0$, $131.5,128.4,127.9,127.6,127.3,123.5,39.5,36.5,20.2$, 20.0; IR (KBr) v: 3483, 2924, 1659, 1117, 1047, 794, 706 $\mathrm{cm}^{-1}$; HRMS-ESI calcd for $\mathrm{C}_{17} \mathrm{H}_{20} \mathrm{NO}[\mathrm{M}+\mathrm{H}]$ 254.1539, found 254.1534.

$N$-Methyl- $N, 2$-di- $p$-tolylacetamide (3dd): Yield $81 \%$ (41.0 mg), light yellow liquid. ${ }^{1} \mathrm{H}$ NMR (500 MHz, $\mathrm{CDCl}_{3}$ ) $\delta: 7.11(\mathrm{~d}, J=8.0 \mathrm{~Hz}, 2 \mathrm{H}), 6.96(\mathrm{~d}, J=7.8 \mathrm{~Hz}, 2 \mathrm{H}), 6.93$ (d, $J=7.8 \mathrm{~Hz}, 2 \mathrm{H}), 6.88$ (d, $J=7.9 \mathrm{~Hz}, 2 \mathrm{H}), 3.32(\mathrm{~s}, 2 \mathrm{H})$, $3.16(\mathrm{~s}, 3 \mathrm{H}), 2.30(\mathrm{~s}, 3 \mathrm{H}), 2.21(\mathrm{~s}, 3 \mathrm{H}) ;{ }^{13} \mathrm{C}$ NMR $(126$ $\left.\mathrm{MHz}, \mathrm{CDCl}_{3}\right) \delta: 170.3,140.5,136.8,135.0,131.4,129.2$, $128.1,127.9,126.3,124.5,39.2,36.6,20.1,20.0$; IR (KBr) $v: 3483,2924,1659,1514,1434,1377,1121,826,780$, $557 \mathrm{~cm}^{-1}$; HRMS-ESI calcd for $\mathrm{C}_{17} \mathrm{H}_{20} \mathrm{NO}[\mathrm{M}+\mathrm{H}]^{+}$ 254.1539, found 254.1533.

$N$-(4-Methoxyphenyl)- $N$-methyl-2-( $p$-tolyl)acetamide (3ed): Yield 89\% (47.9 mg), light yellow liquid. ${ }^{1} \mathrm{H}$ NMR $\left(500 \mathrm{MHz}, \mathrm{CDCl}_{3}\right) \delta: 7.08 \sim 7.00(\mathrm{~m}, 4 \mathrm{H}), 6.96(\mathrm{~d}, J=7.7$ $\mathrm{Hz}, 2 \mathrm{H}), 6.90(\mathrm{~d}, J=8.8 \mathrm{~Hz}, 2 \mathrm{H}), 3.83(\mathrm{~s}, 3 \mathrm{H}), 3.40(\mathrm{~s}$, $2 \mathrm{H}), 3.23(\mathrm{~s}, 3 \mathrm{H}), 2.29(\mathrm{~s}, 3 \mathrm{H}) ;{ }^{13} \mathrm{C} \mathrm{NMR}(126 \mathrm{MHz}$, $\left.\mathrm{CDCl}_{3}\right) \delta: 170.6,157.9,135.8,135.0,131.4,128.0,127.9$, 127.7, 113.7, 54.5, 39.3, 36.7, 20.0; IR (KBr) v: 3483, 2933, 2837, 1651, 1513, 1248, 916, 730, $563 \mathrm{~cm}^{-1}$; HRMS-ESI calcd for $\mathrm{C}_{17} \mathrm{H}_{20} \mathrm{NO}_{2}[\mathrm{M}+\mathrm{H}]^{+} 270.1489$, found 270.1481 .

$N$-(2-Chlorophenyl)- $N$-methyl-2-( $p$-tolyl)acetamide (3fd): Yield 35\% (19.1 mg), light red solid. m.p. 56 $58{ }^{\circ} \mathrm{C}$ (lit. $\left.{ }^{[27]} 66 \sim 67{ }^{\circ} \mathrm{C}\right) ;{ }^{1} \mathrm{H}$ NMR $\left(500 \mathrm{MHz}, \mathrm{CDCl}_{3}\right) \delta$ : $7.43(\mathrm{dd}, J=7.9,1.5 \mathrm{~Hz}, 1 \mathrm{H}), 7.30 \sim 7.17(\mathrm{~m}, 2 \mathrm{H}), 7.08$ (dd, $J=7.6,1.7 \mathrm{~Hz}, 1 \mathrm{H}), 6.96(\mathrm{~d}, J=7.9 \mathrm{~Hz}, 2 \mathrm{H}), 6.85$ (d, $J=8.0 \mathrm{~Hz}, 2 \mathrm{H}), 3.25$ (dd, $J=50.2,14.9 \mathrm{~Hz}, 2 \mathrm{H}), 3.13$ (s, $3 \mathrm{H}), 2.22(\mathrm{~s}, 3 \mathrm{H}) ;{ }^{13} \mathrm{C} \mathrm{NMR}\left(126 \mathrm{MHz}, \mathrm{CDCl}_{3}\right) \delta: 170.2$, $140.1,135.1,132.2,130.8,129.6,129.3,128.6,128.0$, 127.9, 127.1, 39.5, 35.0, 20.0; IR (KBr) v: 2918, 1670, $1480,1370,1125,1063,771,568,466 \mathrm{~cm}^{-1}$.

$N$-(4-Chlorophenyl)- $N$-methyl-2-( $p$-tolyl)acetamide (3gd): Yield 63\% (34.4 mg), light yellow liquid. ${ }^{1} \mathrm{H}$ NMR $\left(500 \mathrm{MHz}, \mathrm{CDCl}_{3}\right) \delta: 7.36(\mathrm{~d}, J=8.5 \mathrm{~Hz}, 2 \mathrm{H}), 7.05(\mathrm{~d}, J=$ $7.8 \mathrm{~Hz}, 4 \mathrm{H}), 6.94(\mathrm{~d}, J=7.2 \mathrm{~Hz}, 2 \mathrm{H}), 3.41(\mathrm{~s}, 2 \mathrm{H}), 3.24(\mathrm{~s}$, $3 \mathrm{H}), 2.30(\mathrm{~s}, 3 \mathrm{H}) ;{ }^{13} \mathrm{C} \mathrm{NMR}\left(126 \mathrm{MHz}, \mathrm{CDCl}_{3}\right) \delta: 171.1$, $142.6,136.3,133.7,132.0,129.9,129.1,129.0,128.8$, 40.6, 37.6, 21.1; IR (KBr) v: 2924, 1660, 1490, 1374, $1120,1014,722,554 \mathrm{~cm}^{-1}$; HRMS-ESI calcd for $\mathrm{C}_{16} \mathrm{H}_{17^{-}}$ CINO $[\mathrm{M}+\mathrm{H}]^{+}$274.0993, found 274.0991.

$N$-Methyl-2-(p-tolyl)- $N$-(4-(trifluoromethyl)phenyl)acetamide (3hd): Yield 57\% (35.0 mg), light yellow liquid. ${ }^{1} \mathrm{H}$ NMR (500 MHz, $\left.\mathrm{CDCl}_{3}\right) \delta: 7.57(\mathrm{~d}, J=8.3 \mathrm{~Hz}, 2 \mathrm{H}), 7.17$ $(\mathrm{d}, J=6.4 \mathrm{~Hz}, 2 \mathrm{H}), 6.97(\mathrm{~d}, J=7.4 \mathrm{~Hz}, 2 \mathrm{H}), 6.86$ (br, 2H), $3.37(\mathrm{~s}, 2 \mathrm{H}), 3.21(\mathrm{~s}, 3 \mathrm{H}), 2.22(\mathrm{~s}, 3 \mathrm{H}) ;{ }^{13} \mathrm{C}$ NMR $(126$ $\left.\mathrm{MHz}, \mathrm{CDCl}_{3}\right) \delta: 169.9,146.1,135.4,130.7,128.2,127.7$, $127.0,125.7,122.7,125.9,123.8,121.6,119.5, \mathrm{q},{ }^{1} J_{\mathrm{C}-\mathrm{F}}=$ $272 \mathrm{~Hz}$ ), 39.7, 36.6, 20.0; IR (KBr) v: 2925, 1664, 1326, $1124,852,612 \mathrm{~cm}^{-1}$; HRMS-ESI calcd for $\mathrm{C}_{17} \mathrm{H}_{17} \mathrm{~F}_{3} \mathrm{NO}$ $[\mathrm{M}+\mathrm{H}]^{+}$308.1257, found 308.1255.

$N$-(4-Cyanophenyl)- $N$-methyl-2-( $p$-tolyl)acetamide (3id): Yield 64\% (33.8 mg), light yellow liquid. ${ }^{1} \mathrm{H}$ NMR $\left(500 \mathrm{MHz} \mathrm{CDCl}_{3}\right) \delta: 7.60(\mathrm{~d}, J=8.4 \mathrm{~Hz}, 2 \mathrm{H}), 7.20 \sim 7.14$ (m, 2H), 6.98 (d, $J=7.7 \mathrm{~Hz}, 2 \mathrm{H}), 6.86(\mathrm{~d}, J=6.3 \mathrm{~Hz}, 2 \mathrm{H})$, $3.42(\mathrm{~s}, 2 \mathrm{H}), 3.21(\mathrm{~s}, 3 \mathrm{H}), 2.23(\mathrm{~s}, 3 \mathrm{H}) ;{ }^{13} \mathrm{C}$ NMR $(126$ $\left.\mathrm{MHz}, \mathrm{CDCl}_{3}\right) \delta: 169.8,146.9,135.5,132.5,130.4,128.3$, $128.2,127.6,127.1,117.0,39.9,36.6,20.0$; IR (KBr) $v$ : 3507, 2924, 2228, 1667, 1505, 1118, 851, $576 \mathrm{~cm}^{-1}$; HRMS-ESI calcd for $\mathrm{C}_{17} \mathrm{H}_{16} \mathrm{~N}_{2} \mathrm{ONa}[\mathrm{M}+\mathrm{Na}]^{+}$287.11548, found 287.11569 .

$N$-Methyl- $N$-(naphthalen-2-yl)-2-( $p$-tolyl)acetamide (3jd): Yield 75\% (43.4 mg), light yellow solid, m.p. 65 $68{ }^{\circ} \mathrm{C} ;{ }^{1} \mathrm{H}$ NMR $\left(500 \mathrm{MHz}, \mathrm{CDCl}_{3}\right) \delta: 7.78(\mathrm{~d}, J=8.6 \mathrm{~Hz}$, $2 \mathrm{H}), 7.71 \sim 7.63(\mathrm{~m}, 1 \mathrm{H}), 7.52 \sim 7.36(\mathrm{~m}, 3 \mathrm{H}), 7.14(\mathrm{~d}, J=$ $6.3 \mathrm{~Hz}, 1 \mathrm{H}), 6.93(\mathrm{~d}, J=7.3 \mathrm{~Hz}, 2 \mathrm{H}), 6.85(\mathrm{~d}, J=6.8 \mathrm{~Hz}$, $2 \mathrm{H}), 3.36(\mathrm{~s}, 2 \mathrm{H}), 3.25(\mathrm{~s}, 3 \mathrm{H}), 2.20(\mathrm{~s}, 3 \mathrm{H}) ;{ }^{13} \mathrm{C} \mathrm{NMR}$ $\left(126 \mathrm{MHz}, \mathrm{CDCl}_{3}\right) \delta: 170.3,140.3,135.1,132.5,131.4$, $131.3,128.8,128.0,127.9,126.8,126.7,125.8,125.7$, 125.1, 124.4, 39.6, 36.6, 20.0; IR (KBr) v: 3053, 2917, 1651, 1504, 1381, 1107, 863, 781, 581, $504 \mathrm{~cm}^{-1}$; HRMSESI calcd for $\mathrm{C}_{20} \mathrm{H}_{19} \mathrm{NONa}[\mathrm{M}+\mathrm{Na}]^{+}$312.13589, found 312.13574.

$N$-Benzyl- $N$-phenyl-2-( $p$-tolyl)acetamide (3kd): Yield $85 \%$ (53.6 mg), light yellow solid. m.p. $71 \sim 74{ }^{\circ} \mathrm{C} ;{ }^{1} \mathrm{H}$ NMR $\left(500 \mathrm{MHz}, \mathrm{CDCl}_{3}\right) \delta: 7.24 \sim 7.18(\mathrm{~m}, 3 \mathrm{H}), 7.18 \sim$ $7.13(\mathrm{~m}, 3 \mathrm{H}), 7.11 \sim 7.07(\mathrm{~m}, 2 \mathrm{H}), 6.96(\mathrm{~d}, J=7.7 \mathrm{~Hz}$, $2 \mathrm{H}), 6.87(\mathrm{~d}, J=7.7 \mathrm{~Hz}, 2 \mathrm{H}), 6.85 \sim 6.79(\mathrm{~m}, 2 \mathrm{H}), 4.80(\mathrm{~s}$, 2H), $3.33(\mathrm{~s}, 2 \mathrm{H}), 2.22(\mathrm{~s}, 3 \mathrm{H}) ;{ }^{13} \mathrm{C} \mathrm{NMR}(126 \mathrm{MHz}$, $\left.\mathrm{CDCl}_{3}\right) \delta: 170.0,141.2,136.4,135.1,131.3,128.4,128.0$, 
$127.93,127.85,127.7,127.3,127.0,126.3,52.2,39.7$, 20.0; IR (KBr) v: 2980, 1642, 1596, 1494, 1401, 1297, $768,709 \mathrm{~cm}^{-1}$. HRMS-ESI calcd for $\mathrm{C}_{22} \mathrm{H}_{21} \mathrm{NONa}[\mathrm{M}+$ $\mathrm{Na}^{+} \quad 338.15154$, found 338.15109 .

$N$-Cyclohexyl- $N$-phenyl-2-( $p$-tolyl)acetamide $\quad$ (3ld): Yield 80\% (49.1 mg), yellow solid. m.p. 68 70 ${ }^{\circ} \mathrm{C} ;{ }^{1} \mathrm{H}$ NMR $\left(500 \mathrm{MHz}, \mathrm{CDCl}_{3}\right) \delta: 7.41 \sim 7.30(\mathrm{~m}, 3 \mathrm{H}), 7.07 \sim$ $6.98(\mathrm{~m}, 4 \mathrm{H}), 6.91(\mathrm{~d}, J=7.9 \mathrm{~Hz}, 2 \mathrm{H}), 4.61(\mathrm{tt}, J=12.1$, $3.5 \mathrm{~Hz}, 1 \mathrm{H}), 3.26(\mathrm{~s}, 2 \mathrm{H}), 2.29(\mathrm{~s}, 3 \mathrm{H}), 1.82(\mathrm{~d}, J=11.5$ $\mathrm{Hz}, 2 \mathrm{H}), 1.70$ (d, $J=13.6 \mathrm{~Hz}, 2 \mathrm{H}), 1.55$ (d, $J=13.1 \mathrm{~Hz}$, 1H), 1.38 (qt, $J=13.2,3.3 \mathrm{~Hz}, 2 \mathrm{H}), 1.01$ (qd, $J=12.4,3.5$ $\mathrm{Hz}, 2 \mathrm{H}), 0.89$ (qt, $J=13.1,3.7 \mathrm{~Hz}, 1 \mathrm{H}) ;{ }^{13} \mathrm{C}$ NMR $(126$ $\left.\mathrm{MHz}, \mathrm{CDCl}_{3}\right) \delta: 170.5,139.1,135.9,132.6,130.7,129.0$, $128.9,128.2,54.3,41.5,31.6,25.8,25.4,21.1$; IR (KBr) $v$ : 2926, 2853, 1655, 1592, 1492, 1258, 1067, 767, 713, 486 $\mathrm{cm}^{-1}$; HRMS-ESI calcd for $\mathrm{C}_{21} \mathrm{H}_{26} \mathrm{NO}[\mathrm{M}+\mathrm{H}]^{+}$ 308.2009, found 308.2001.

$N, N$-Diphenyl-2-( $p$-tolyl)acetamide (3md): Yield 43\% (25.9 mg), yellow solid. m.p. $72 \sim 76{ }^{\circ} \mathrm{C}$ (m.p. ${ }^{[28]} 74 \sim$ $\left.77{ }^{\circ} \mathrm{C}\right) ;{ }^{1} \mathrm{H}$ NMR $\left(500 \mathrm{MHz}, \mathrm{CDCl}_{3}\right) \delta: 7.34$ (br, 5H), $7.25 \sim 7.12(\mathrm{~m}, 5 \mathrm{H}), 7.07(\mathrm{~d}, J=7.9 \mathrm{~Hz}, 2 \mathrm{H}), 7.01(\mathrm{~d}, J=$ $7.9 \mathrm{~Hz}, 2 \mathrm{H}), 3.61(\mathrm{~s}, 2 \mathrm{H}), 2.32(\mathrm{~s}, 3 \mathrm{H}) ;{ }^{13} \mathrm{C}$ NMR $(126$ $\left.\mathrm{MHz}, \mathrm{CDCl}_{3}\right) \delta: 170.2,141.8,135.2,131.0,128.4,128.0$, $127.9,126.8,125.3,40.7,20.0$; IR (KBr) v: 2935, 1671, $1589,1352,1308,1279,1148,805,759,703,581,490$ $\mathrm{cm}^{-1}$.

$N$-Methyl- $N$-phenylacetamide (3na): Yield $68 \%(20.3$ mg), light red solid. m.p. 100 $102{ }^{\circ} \mathrm{C} ;{ }^{1} \mathrm{H}$ NMR (500 $\left.\mathrm{MHz}, \mathrm{CDCl}_{3}\right) \delta: 7.41(\mathrm{t}, J=7.5 \mathrm{~Hz}, 2 \mathrm{H}), 7.32(\mathrm{t}, J=7.2$ $\mathrm{Hz}, 1 \mathrm{H}), 7.18$ (d, J=7.6 Hz, 2H), 3.25 (s, 3H), 1.86 (s, $3 \mathrm{H}) ;{ }^{13} \mathrm{C} \mathrm{NMR}\left(126 \mathrm{MHz}, \mathrm{CDCl}_{3}\right) \delta: 169.5,143.6,128.7$, 126.7, 126.1, 36.1, 21.4; IR (KBr) v: 3046, 1656, 1598, 1496, 1297, 707, $559 \mathrm{~cm}^{-1}$; HRMS-ESI calcd for $\mathrm{C}_{9} \mathrm{H}_{11} \mathrm{NONa}[\mathrm{M}+\mathrm{Na}]^{+}$172.07329, found 172.07333 .

Morpholino(phenyl)methanone (3ol): Yield 76\% (29.0 mg), white solid, m.p. $73 \sim 74{ }^{\circ} \mathrm{C} ;{ }^{1} \mathrm{H}$ NMR $(500 \mathrm{MHz}$, $\left.\mathrm{CDCl}_{3}\right) \delta: 7.40 \sim 7.28(\mathrm{~m}, 5 \mathrm{H}), 4.06 \sim 3.10(\mathrm{~m}, 8 \mathrm{H}) ;{ }^{13} \mathrm{C}$ NMR $\left(126 \mathrm{MHz}, \mathrm{CDCl}_{3}\right) \delta: 169.41,134.30,128.86$, 127.54, 126.07, 65.87; IR (KBr) v: 3414, 1621, 1436, 1274, 1109, 1012, 731, $709 \mathrm{~cm}^{-1}$; HRMS-ESI calcd for $\mathrm{C}_{11} \mathrm{H}_{13} \mathrm{NO}_{2} \mathrm{Na}[\mathrm{M}+\mathrm{Na}]^{+}$214.08385, found 214.08389.

Supporting Information ${ }^{1} \mathrm{H}$ NMR and ${ }^{13} \mathrm{C}$ NMR spectra of 3aa $\sim 3 \mathbf{a o}, 3 \mathbf{b d} \sim \mathbf{3 m d}$, 3na, 3ol and HRMS spectra of 3ac, 3ae $\sim$ 3ag, 3ai, 3aj, 3bd $\sim$ 3ed, 3gd $\sim$ 3ld, 3na, 3ol are available free of charge via the Internet at http://siocjournal.cn/.

\section{References}

[1] (a) Valeur, E.; Bradley, M. Chem. Soc. Rev. 2009, 38, 606. (b) de Figueiredo, R. M.; Suppo, J.-S.; Campagne, J.-M. Chem. Rev. 2016, 116, 12029.

[2] Roy, S.; Roy, S.; Gribble, G. W. Tetrahedron 2012, 68, 9867.

[3] Organotin reagents, see:

(a) Balas, L.; Jousseaume, B.; Shin, H.; Verlhac, J.-B.; Wallian, F. Organometallics 1991, 10, 366.

(b) Jousseaume, B.; Kwon, H.; Verlhac, J.-B.; Denat, F.; Dubac, J.
Synlett 1993, 117.

(c) Murakami, M.; Hoshino, Y.; Ito, H.; Ito, Y. Chem. Lett. 1998 , 163.

(d) Hu, W.; Zheng, J.; Li, M.; Wu, W.; Liu, H.; Jiang, H. Chin. J. Chem. 2018, 36, 712.

[4] Lemoucheux, L.; Rouden, J.; Lasne, M.-C. Tetrahedron Lett. 2000 , 41, 9997.

[5] Rieke, R. D.; Kim, S.-H. Tetrahedron Lett. 2012, 53, 3478.

[6] Lysén, M.; Kelleher, S.; Begtrup, M.; Kristensen, J. L. J. Org. Chem. 2005, 70, 5342.

[7] Duan, Y.-Z.; Deng, M.-Z. Synlett 2005, 355.

[8] Yasui, Y.; Tsuchida, S.; Miyabe, H.; Takemoto, Y. J. Org. Chem. 2007, 72, 5898.

[9] Krishnamoorthy, R.; Lam, S. Q.; Manley, C. M.; Herr, R. J. J. Org. Chem. 2010, 75, 1251 .

[10] Kochi, T.; Urano, S.; Seki, H.; Mizushima, E.; Sato, M.; Kakiuchi, F. J. Am. Chem. Soc. 2009, 131, 2792.

[11] (a) Matsuzono, M.; Fukuda, T.; Iwao, M. Tetrahedron Lett. 2001, 42,7621 .

(b) Chao, W.-R.; Yean, D.; Amin, K.; Green, C.; Jong, L. J. Med. Chem. 2007, 50, 3412.

[12] Lemoucheux, L.; Seitz, T.; Rouden, J.; Lasne, M.-C. Org. Lett. 2004, 6, 3703.

[13] Selected reviews, see:

(a) Baudoin, O. Angew. Chem., Int. Ed. 2007, 46, 1373.

(b) Gooßen, L. J.; Rodriguez, N.; Gooßen, K. Angew. Chem., Int. Ed. 2008, 47, 3100 .

(c) Rodríguez, N.; Gooßen, L. J. Chem. Soc. Rev. 2011, 40, 5030. (d) Dzik, W. I.; Lange, P. P.; Gooßen, L. J. Chem. Sci. 2012, 3, 2671.

(e) Wei, Y.; Hu, P.; Zhang, M.; Su, W. Chem. Rev. 2017, 117, 8864.

[14] Selected examples, see:

(a) Myers, A. G.; Tanaka, D.; Mannion, M. R. J. Am. Chem. Soc. 2002, 124, 11250 .

(b) Tanaka, D.; Romeril, S. P.; Myers, A. G. J. Am. Chem. Soc. 2005, 127, 10323 .

[15] Gooßen, L. J.; Deng, G.; Levy, L. M. Science 2006, 313, 662.

[16] (a) Gooßen, L. J.; Linder, C.; Rodriguez, N.; Lange, P. P.; Fromm, A. Chem. Commun. 2009, 7173.

(b) Cornella, J.; Sanchez, C.; Banawa, D.; Larrosa, I. Chem. Commun. 2009, 7176.

[17] (a) Cornella, J.; Rosillo-Lopez, M.; Larrosa, I. Adv. Synth. Catal. 2011, 353, 1359 .

(b) Dupuy, S.; Lazreg, F.; Slawin, A. M. Z.; Cazin, C. S. J.; Nolan, S. P. Chem. Commun. 2011, 47, 5455.

[18] Sun, Z.-M.; Zhao, P. Angew. Chem., Int. Ed. 2009, 48, 6726.

[19] Shang, R.; Fu, Y.; Wang, Y.; Xu, Q.; Yu, H.-Z.; Liu, L. Angew. Chem., Int. Ed. 2009, 48, 9350.

[20] (a) Jiao, J.; Zhang, X.-R.; Chang, N.-H.; Wang, J.; Wei, J.-F.; Shi, X.-Y.; Chen, Z.-G. J. Org. Chem. 2011, 76, 1180.

(b) Iwai, T.; Fujihara, T.; Terao, J.; Tsuji, Y. J. Am. Chem. Soc. 2010, 132, 9602.

[21] Yamasaki, R.; Morita, K.; Iizumi, H.; Ito, A.; Fukuda, K.; Okamoto, I. Chem.-Eur. J. 2019, 25, 10118.

[22] Allah, T. N.; ne Savourey, S.; Berthet, J.-C.; Nicolas, E.; Cantat, T. Angew. Chem., Int. Ed. 2019, 58, 10884.

[23] Song, G.; Sun, G.; Tang, Y.; Mai, W. J. Chem. Res. 2013, 630.

[24] Zhou, Y.; Zhang, X.; Zhang, Y.; Ruan, L.; Zhang, J.; Zhang-Negrerie, D.; Du, Y. Org. Lett. 2017, 19, 150.

[25] Zhang, Z.; Liu, Y.-H.; Zhang, X.; Wang, X.-C. Tetrahedron 2019, $75,2763$.

[26] Bao, Y.-S.; Zhaorigetu, B.; Agula, B.; Baiyin, M.; Jia, M. J. Org. Chem. 2014, 79, 803.

[27] Ackermann, L.; Vicente, R.; Hofmann, N. Org. Lett. 2009, 11, 4274.

[28] Das, K. G.; Funke, P. T.; Bose, A. K. J. Am. Chem. Soc. 1964, 86, 3729.

(Cheng, F.) 